\title{
Towards a fully automated eclipsing binary solver for Gaia
}

\author{
Brandon Tingley, Gilles Sadowski \& Christos Siopis \\ Institut d'Astronomie et d'Astrophysique \\ Université Libre de Bruxelles \\ CP226 \\ Boulevard du Triomphe \\ B-1050 Brussels \\ Belgium \\ email: btingley@ulb.ac.be, gsadowski@ulb.ac.be, csiopis@ulb.ac.be
}

\begin{abstract}
Gaia, an ESA cornerstone mission, will obtain of the order of 100 high-precision photometric observations and lower precision radial velocity measurements over five years for around a billion stars - several hundred thousand of which will be eclipsing binaries. In order to extract the characteristics of these systems, a fully automated code must be available. During the process of this development, two tools that may be of use to the transit community have emerged: a very fast, simple, detached eclipsing binary simulator/solver based on a new approach and an interacting eclipsing binary simulator with most of the features of the Wilson-Devinney and Nightfall codes, but fully documented and written in easy-to-follow and highly portable Java. Currently undergoing development and testing, this code includes an intuitive graphical interface and an optimizer for the estimation of the physical parameters of the system.
\end{abstract}

Eclipsing binaries are very important objects, as they help us understand the nature and evolution of stars. With photometry and radial velocities, it is possible to extract the physical parameters of the components to a precision limited only by our ability to model the light from these objects. Gaia will be a very important tool in this effort, gathering the required data for tens of thousands eclipsing binaries. However, this does leave the sizable challenge of having to analyze all of these time series, as even the analysis of a single system is very complex. As such, we have decided to use the following approach to the problem: a simple, fast detached binary solver extracts parameters from just the photometry, operating under the often-poor assumption that the system is detached. These parameters are fed to a global optimizer, which uses them as a starting point in finding a solution using a fully developed simulator that includes the interactive aspects of binary stars (mutual irradiation and gravitational distortion) that affect the light curves.

The GeSSS simulator is such a fully developed simulator. It represents a binary system with Roche model assumption: the form of the surface of each star depends on the gravitational potential generated by two points masses revolving around the center of the mass of the system. Because the Java programming language was selected for all Gaia data processing, we had to write a simulator code from the ground up. The main algorithms were largely inspired by the Nightfall software but the overall code design has evolved significantly. Some important details of the algorithms, such as mutual irradiation and partial visibility were modified, leading to a few minor difference in the synthetic light curve, as shown in the accompanying figures. The code is still under development and a thorough validation of the science is still in progress. The goal is to compare GeSSS with the "benchmark" code of Wilson-Devinney and modify the GeSSS components 
accordingly, wherever needed. We have also developed a very basic graphical user interface, primarily intended for qualitative testing.

The fast, simple detached binary simulator has three aspects that make is different than other similar codes found in the literature. First, the equation describe the time between eclipses has been truly solved for the first time, replacing an admittedly very good approximation. Secondly, equation were derived that demonstrated that the luminosities of the star in binary eclipses could be separated from the other parameters, modeling eclipsing between luminous and non-luminous bodies, rather than two luminous bodies. This enables the third difference: rather than performing the full eclipsing modeling while creating the light curves, it becomes possible to create all the necessary models a priori: fully limb-darkened eclipses can be calculated in advance with just two parameters (impact parameter and ratio of radii) and store, then very accurate light curves can be constructed from this small library of models by choosing the appropriate durations, eclipses centers, impact parameters, ratio of radii, and luminosity ratio, as can been seen below.
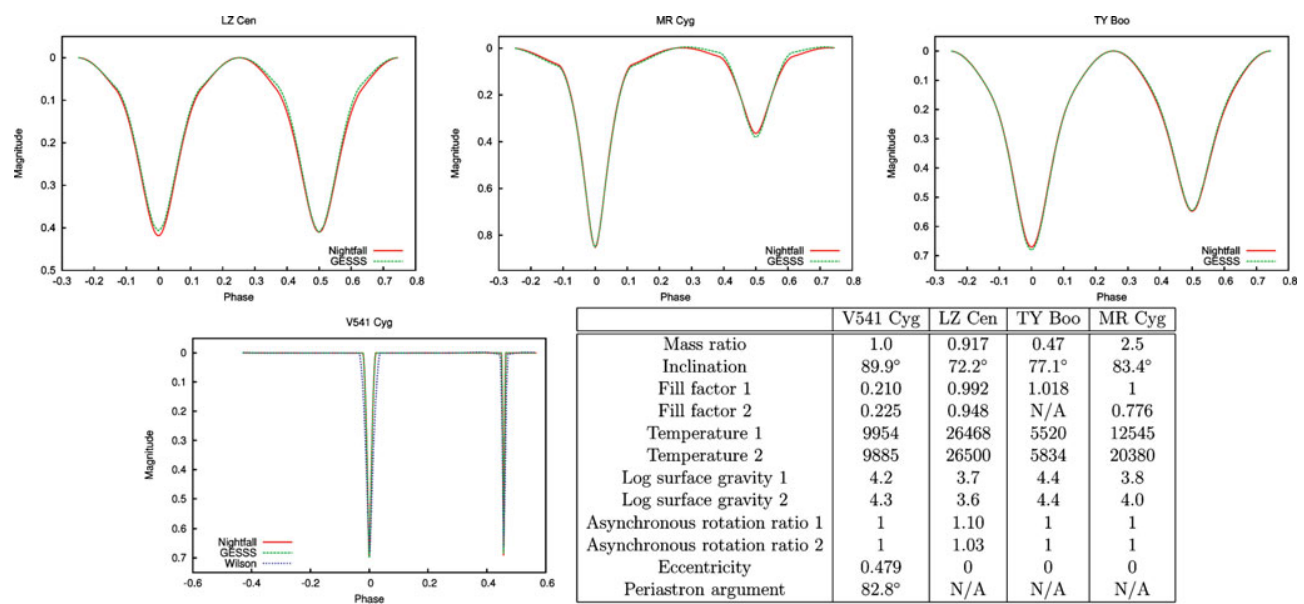

\begin{tabular}{|c|c|c|c|c|}
\hline \multicolumn{5}{|c|}{ Phase } \\
\hline \hline Mass ratio & V54 Cyg & LZ Cen & TY Boo & MR Cyg \\
\hline \hline Inclination & 1.0 & 0.917 & 0.47 & 2.5 \\
Fill factor 1 & $89.9^{\circ}$ & $72.2^{\circ}$ & $77.1^{\circ}$ & $83.4^{\circ}$ \\
Fill factor 2 & 0.210 & 0.992 & 1.018 & 1 \\
Temperature 1 & 0.225 & 0.948 & N/A & 0.776 \\
Temperature 2 & 9954 & 26468 & 5520 & 12545 \\
Log surface gravity 1 & 9885 & 26500 & 5834 & 20380 \\
Log surface gravity 2 & 4.2 & 3.7 & 4.4 & 3.8 \\
Asynchronous rotation ratio 1 & 1 & 3.6 & 4.4 & 4.0 \\
Asynchronous rotation ratio 2 & 1 & 1.10 & 1 & 1 \\
Eccentricity & 0.479 & 0 & 1 & 1 \\
Periastron argument & $82.8^{\circ}$ & N/A & N/A & N/A \\
\hline
\end{tabular}
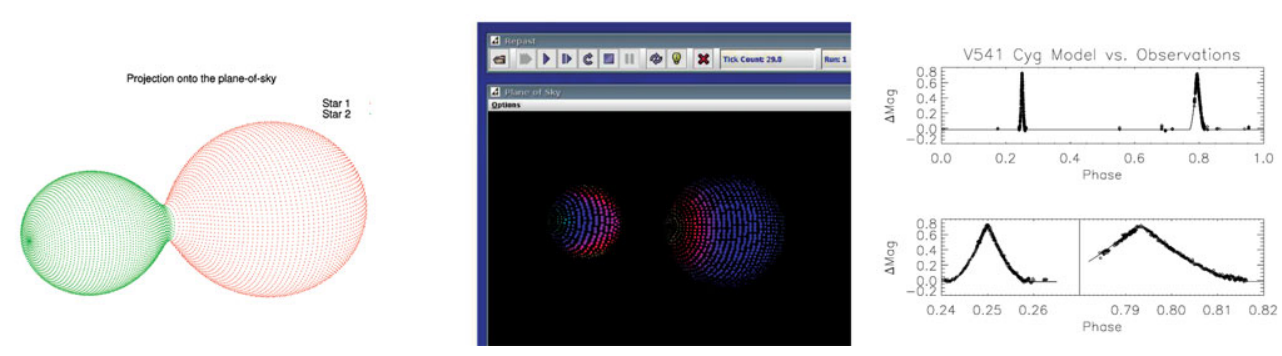

Figure 1. The figures in the top row and middle row-left show comparisons of models created by Nightfall and GeSSS for the stars LZ Cen, MR Cyg, LY Boo, and v541 Cyg, with a Wilson-Devinney model included in the last. The parameters used are in the middle-right figure. The bottom row left and middle show the graphical interface of the GeSSS simulator and the bottom right a sample model created by the fast simple detached binary modeler for the elliptical, detached system v541 Cyg using parameters from the the literature derived using the Wilson-Devinney code. 